Gut, 1982, 23, 150-156

\title{
Motor function of gastric antrum and pylorus for evacuation of low and high viscosity meals in dogs
}

\author{
J PRÖVE AND H-J EHRLEIN* \\ From the Institute of Zoophysiology, University of Hohenheim, Stuttgart, FRG
}

SUMMARY In five conscious dogs motility of the antrum, pyloric sphincter, and duodenum was recorded with strain gauge transducers and induction coils. Gastric evacuation of low, medium, and high viscosity meals was measured via a duodenal cannula and observed simultaneously by radiography. Computer analysis of the propagation of the gastric waves revealed increased velocity in the distal antrum but no simultaneous contractions of the terminal antrum and pyloric sphincter. Radiography showed, and measurements of the antral diameter confirmed, that the indentations of the gastric waves were significantly deeper with the low viscosity liquid meal compared with the medium and high viscosity meals. Thereby, retropulsion of the medium and high viscosity ingesta was produced. Results indicated that gastric evacuation was regulated predominantly by the depth of the peristaltic indentation, which depended on the viscosity of the gastric contents. Nothing indicated that the phasic contractions of the pyloric sphincter were of importance for the regulation of gastric emptying.

In previous studies on rabbits ${ }^{1}$ it was found that gastric evacuation depended on the viscosity of the test meals. These findings were in agreement with the results of other groups which have measured gastric emptying of solid particles and of liquids labelled with isotopes. ${ }^{3-7}$ However, little is known about the different mechanisms involved in the gastric emptying of liquids and solids. Dozois et al. ${ }^{8}$ and Wilbur and Kelly' studied the effect of antrectomy and vagotomy on gastric emptying of liquids and of radiopaque plastic spheres in dogs. They concluded that the gastric emptying of liquids is regulated by the proximal stomach, whereas that of solids is regulated by the distal stomach. The simultaneous contractions of the terminal antrum and of the pyloric sphincter produced retropulsion as described in dogs ${ }^{10}$ and in humans. ${ }^{11-13}$ Thereby, the plastic spheres were retained in the stomach, while liquids were emptied very quickly. These results support the idea of Cannon ${ }^{14}$ that the pyloric sphincter is the 'keeper of the gate'. However, it is doubtful whether the evacuation of large plastic spheres represents the emptying pattern of normal food. Moreover, these results are largely based on fluoroscopic observations. Exact measurements of

*Address for correspondence: $\mathrm{Dr} \mathrm{H}-\mathrm{J}$ Ehrlein, Institut für Zoophysiologie, Universität Hohenheim, Garbenstr. 30, D-7000 Stuttgart 70, FRG.

Received for publication 8 July 1981 the mechanical activity of the terminal antrum have not yet been carried out, and it is not known if this mechanism is also responsible for the regulation of gastric emptying of ordinary food.

Our objective was to clarify the mechanism by which gastric evacuation of liquid and visc us ingesta is regulated. This was accomplished by simultaneous radiography, recording the gastric and duodenal motility, and measuring the gastric evacuation.

The study was part of a dissertation. ${ }^{15}$ An abstract of the results was presented at the 7th International Symposium on Gastrointestinal Motility. ${ }^{16}$

\section{Methods}

Five conscious male dogs were studied. Under general anaesthesia extraluminal strain gauge transducers were sutured to the circular axis of the gastric antrum, pyloric sphincter, and duodenum (Fig. 1). The transducers were positioned on the duodenum $2.5 \mathrm{~cm}$ distal and on the antrum $2.5 \mathrm{~cm}$ (A1), $5 \mathrm{~cm}$ (A2) and $7.5 \mathrm{~cm}$ (A3) proximal to the gastroduodenal junction. The transducers were prepared as previously described. ${ }^{17}$ In two dogs the external diameter of the pylorus was measured with induction coils which were sutured to the serosal surface on opposite sides of the pyloric sphincter. In two other dogs induction coils were sutured to the 


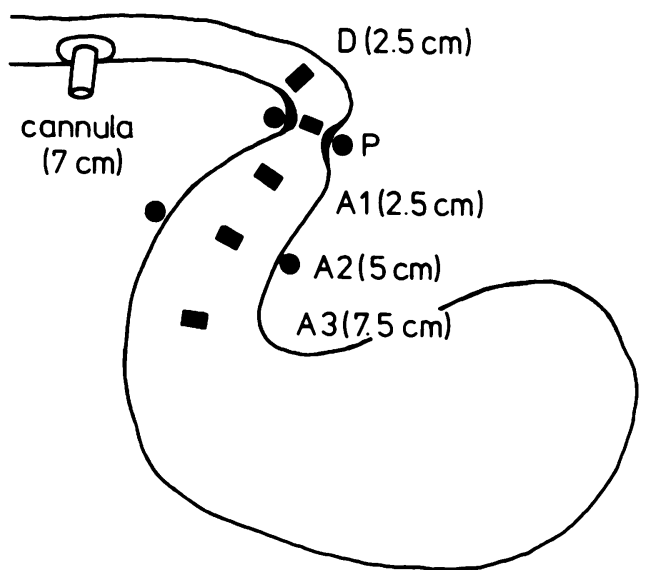

Fig. 1 Position of strain gauge transducers ( $\square)$, induction coils (๑), and duodenal cannula on antrum (A1,A2, A3), pyloric sphincter $(P)$, and duodenum $(D)$. Centimetres indicate distances to pyloric sphincter.

antrum at site A2 (Fig. 1). Induction coils were used because the diameter provides better information about the opening and closure of the pylorus as well as the depth of the peristaltic contractions at site A2 than do the recordings with strain gauges. The application of induction coils for recording the activity of the pyloric sphincter was described by Brody and Quigley ${ }^{18}$ and by Louckes et al. ${ }^{19}$ The induction coils and the measuring device were constructed as described by Engelhardt et al. ${ }^{20}$

Gastric emptying was measured by a silicone cannula placed into the duodenum $7 \mathrm{~cm}$ distal to the gastroduodenal junction (Fig. 1).

TEST MEALS

Three test meals were used. All were $200 \mathrm{ml}$ and consisted of saline solution $(19.6 \mathrm{~g} / 1$ trisodium citrate, $\mathrm{pH} 7 \cdot 8$, osmolality $150 \mathrm{mOsm} / 1)$ mixed with $150 \mathrm{~g}$ barium sulphate. Meals of medium viscosity (2.3-8 $\times 10^{5}$ centipose) and high viscosity (1.1-3.6 $\times 10^{6}$ centipose) were prepared by stirring in $30 \mathrm{~g}$ or $40 \mathrm{~g}$ potato granules.

\section{PROCEDURES}

Studies were begun 10 days after each operation. The dogs were fasted for 18 hours. During the experiments they were made to stand on a special rack and were positioned in a hammock. One of the test meals was administered into the stomach via a gastric tube, when phase II of the interdigestive activity was evident. ${ }^{21}$ Gastric emptying was measured by collecting the effluent of the duodenal cannula. The concentration of chloride was measured in the liquid portion of each sample in order to calculate the amount of gastric juice.

\section{MOTILITY RECORDS}

Motor activities of the gastric antrum, pyloric sphincter, and duodenum were recorded simultaneously with the measurement of gastric emptying. The signals of the induction coils were calibrated at the beginning of each experiment using a second pair of induction coils which were constructed with the same technical measurements as the implanted ones. The strain gauge transducers were calibrated indirectly, in that their signals were compared with a definite strain which was produced by an electronic device (Elan, Düsseldorf, FRG). The recordings were made on a multichannel recorder and on magnetic tape via frequency modulation.

\section{ANALYSIS}

The recordings of motility were analysed with a computer. Details of the computer analysis were described previously. ${ }^{22}$ The amplitudes of contractions, the contraction-time (the time from the beginning to the maximum of the contraction), the contractile intervals (intervals between the beginning of two successive contractions), and the timedifferences between the contraction maxima of two adjacent recording sites were evaluated.

\section{RADIOGRAPHY}

The movement of the ingesta within the stomach and the transfer into the duodenum were observed fluoroscopically using an $x$-ray image intensifier video system (Siemens, Erlangen, FRG). To correlate the transducer recordings with fluoroscopy the motility tracings were displayed on the monitor and on the video-tape by a second television camera and a mixer. Analysis was made from the video recordings using slow, motion and still-image play back. The advantages of this method have been previously described. ${ }^{21}$

\section{STATISTICS}

The significance of the differences in the diameter of the antrum at site A2 was tested by Student's $t$ test. The U-test of Wilcoxon, Mann, and Whitney ${ }^{23}$ was used to test the differences in the distribution of amplitudes and the time-differences between contractions of two adjacent recording sites with various test meals.

\section{Results}

\section{GASTRIC MOTILITY AND EMPTYING}

After the injection of the various test meals during phase II of the interdigestive activity, the irregular motility of the empty stomach changed to regular patterns of the digestive activity (Fig. 2). Gastric 

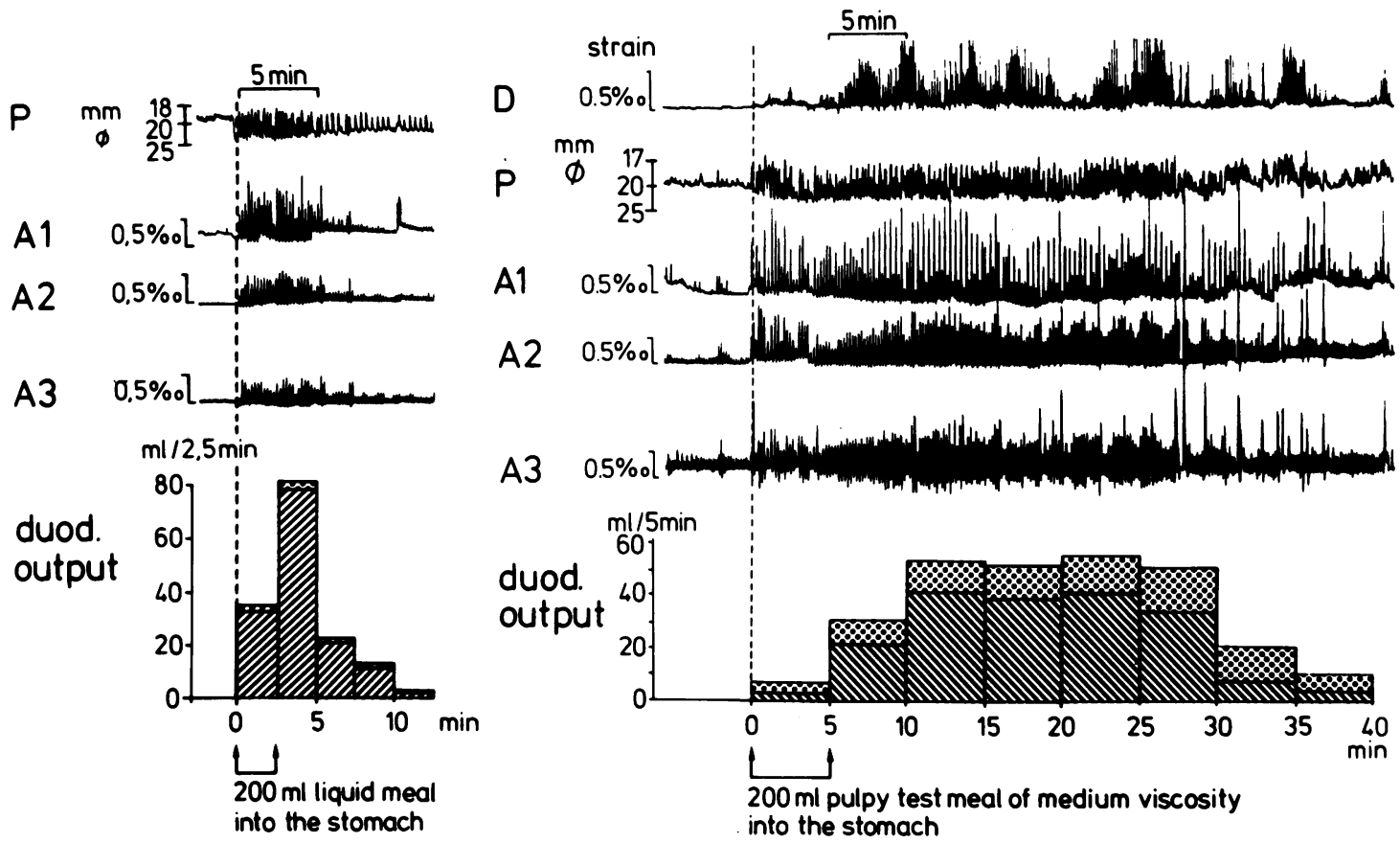

Fig. 2 Gastric and duodenal motility and gastric emptying after instillation of low viscosity liquid meal (left panel) and medium viscosity meal (right panel) into stomach. Location of transducers as in Fig. 1. Dotted columns represent gastric juice, hatched columns represent original test meal.

evacuation increased immediately during the injection of the low viscosity liquid meal, whereas gastric evacuation increased only gradually with the test meals of medium and high viscosity. The periods of gastric evacuation and the digestive pattern of motor activity lasted $28.7 \pm 7.3 \mathrm{~min}$ with the low viscosity meal ( 20 experiments), $64.8 \pm 17.5$ min with the medium viscosity meal (21 experiments), and $95.3 \pm 18.7 \mathrm{~min}$ with the high viscosity meal ( 15 experiments).

\section{RADIOGRAPHIC OBSERVATIONS}

The simultaneous observations of the motility recordings and the movements of the ingesta revealed marked differences in the patterns of gastric evacuation depending on the viscosity of the ingesta. The pylorus opened and closed in the rhythm of the antral peristalic waves. The sphincter relaxed when the peristaltic wave travelled over the middle of the antrum (Fig. 3, I and II). The indentations of the antral waves became deep in the case of the low viscosity liquid contents; thus all the liquid in front of the contraction was discharged through the small opening of the relaxed pylorus into the duodenum. There were lesser peristaltic indentations with the meals of medium and high viscosity. As the central opening of the antral
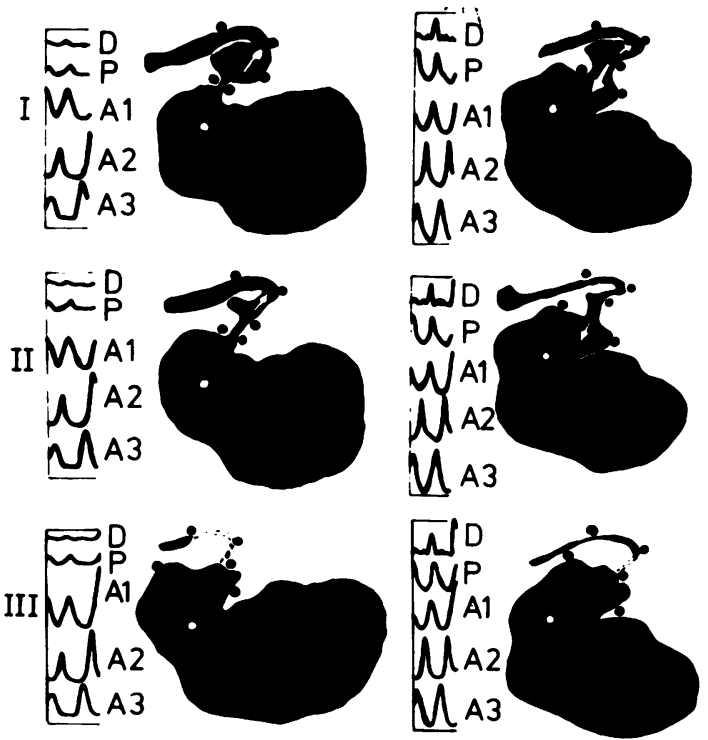

low viscosity liquid meal

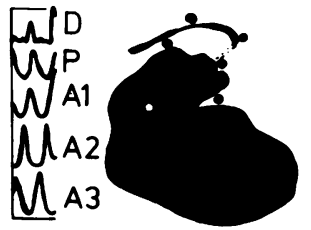

medium viscosity meal

Fig. 3 Stomach outline and transducer recordings showing evacuation of low viscosity liquid contents by deep peristaltic indentations and retropulsion of medium viscosity meal. Location of transducers as in Fig. 1. 
contraction remained larger than that of the relaxed pyloric sphincter, most of the viscous ingesta were retropelled into the relaxing proximal antrum; only small amounts of the ingesta which were sufficiently liquefied were delivered into the duodenum (Fig. 3, I and II). When the peristaltic wave proceeded over the terminal antrum, the pyloric sphincter just started to contract (Fig. 3, III). Whereas the residues of the liquid and sufficiently diluted ingesta could escape through the pylorus into the duodenum, viscous ingesta were retropelled into the proximal antrum. Thus, the evacuation of liquid and viscous ingesta was different; it was regulated by adjusting the depth of the peristaltic indentation in relation to the small opening of the pylorus.

When the pulpy ingesta were liquefied by gastric juice the indentations of the antral waves became deeper, gastric evacuation increased, and retropulsion decreased. Sometimes, during the contractions of the terminal antrum, the flow of ingesta into the duodenum was interrupted by contractions of the duodenal bulb which had often started before the pylorus was closed.

\section{ANALYSIS OF GASTRIC MOTILITY \\ Amplitude of contractions}

As shown in Fig. 2, gastric and duodenal motility was stimulated when the test meals entered the stomach. With all test meals the frequency of contractions was maximal-that is, one contraction followed the other without interruption. Computer analysis of 6935 contractions in five dogs revealed a mean contractile interval of $11.7 \pm 1.4 \mathrm{~s}$; this corresponds to a mean frequency of 5.1 contractions/min. As radiography showed that the depth of the antral indentations was different with the various test meals, the amplitudes of the contractions at site A2 were analysed in five-minute periods. The amplitudes of the contractions of site A2 which were recorded with strain gauge transducers were not significantly different between the low, medium and high viscosity meals. When, however, the diameter of site A2 was recorded with induction coils, the depth of the antral indentation during maximal contraction (Fig. 4, upper lines) was deeper with the low viscosity liquid meal than with those of medium and high viscosity. When the medium and high viscosity meals were liquefied by gastric juice during the course of the experiments the depth of the antral indentation increased. For statistical analysis of this result, comparisons were made between the data taken during the first 10 minutes with the low viscosity meal, the first 20 minutes with the meal of medium viscosity, and the first 30 minutes with the meal of high viscosity.

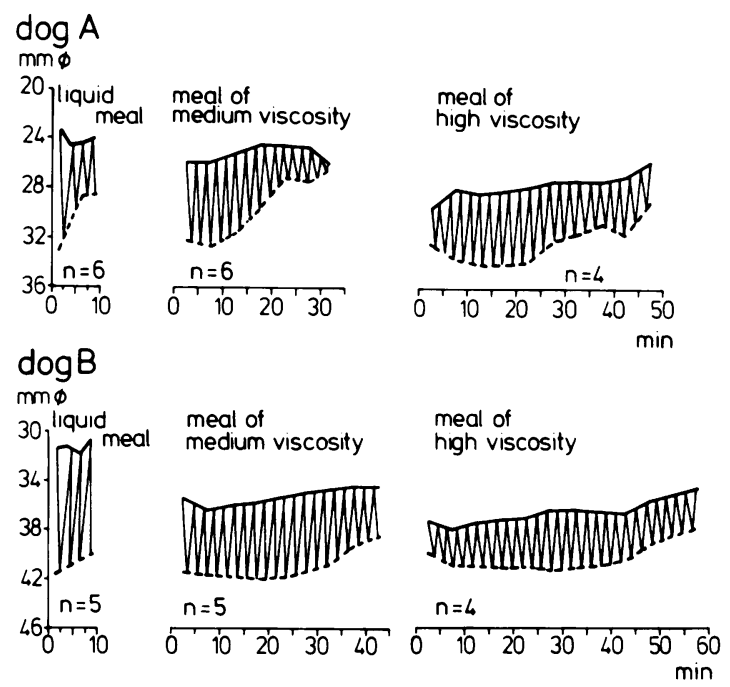

Fig. 4 External diameter of antrum at site $A 2(5 \mathrm{~cm}$ proximal to pyloric sphincter) with liquid and pulpy test meals in two dogs analysed in five minute periods. Upper lines indicate mean values during contraction maxima. Lower dotted lines indicate mean values during contraction minima. $n$ : number of experiments.

The results (Table 1) confirmed that the depth of the antral indentation was significantly deeper with the low viscosity liquid meal compared with the meals of medium and high viscosity.

\section{Propagation of antral contractions}

The propagation of the peristaltic wave from site A 3 of the proximal antrum to the pyloric sphincter is shown in Fig. 5 (left panel). To evaluate the propagation of the peristaltic waves, the timedifferences of contraction maxima between sites A2 and $A 1$ and between site $A 1$ and the pyloric sphincter were analysed by the computer. As no significant differences of the data were found between the various test meals or by dilution of the medium and high viscosity meals, the data of all

Table 1 External diameter of middle of antrum (site A2) during contraction maxima of peristaltic waves

\begin{tabular}{|c|c|c|c|c|c|c|}
\hline \multirow[t]{2}{*}{ Dog } & \multicolumn{3}{|c|}{ Viscosity of test meal } & \multicolumn{3}{|c|}{ Significance } \\
\hline & $\begin{array}{l}\text { Low } \\
0-10 \text { min }^{*} \\
1 \\
\left(\mathrm{~mm}^{* *}\right)\end{array}$ & $\begin{array}{l}\text { Medium } \\
0-20 \text { min }^{*} \\
2 \\
\left(\mathrm{~mm}^{* *}\right)\end{array}$ & $\begin{array}{l}\text { High } \\
0-30 \text { min }^{*} \\
3 \\
\left(\mathrm{~mm}^{* *}\right)\end{array}$ & $1 / 2$ & $2 / 3$ & $1 / 3$ \\
\hline $\begin{array}{l}\mathbf{A} \\
\mathbf{B}\end{array}$ & $\begin{array}{l}24.0 \pm 0.8(6) \\
31.4 \pm 1 \cdot 1(5)\end{array}$ & $\begin{array}{l}25.3 \pm 1.5(6) \\
35.9 \pm 1.3(5)\end{array}$ & $\begin{array}{l}28.2 \pm 1.4(4) \\
37.3 \pm 1.4(4)\end{array}$ & $\begin{array}{l}\dagger \\
\ddagger\end{array}$ & \pm & $\begin{array}{l}\ddagger \\
\ddagger\end{array}$ \\
\hline
\end{tabular}

Data are means $\pm S D$. Numbers of tests are indicated in parentheses. *Time of analysis after administration of the test meal.

$+\mathrm{P}<0.05 ; \neq \mathrm{P}<0.001 ; * *$ diameter. 


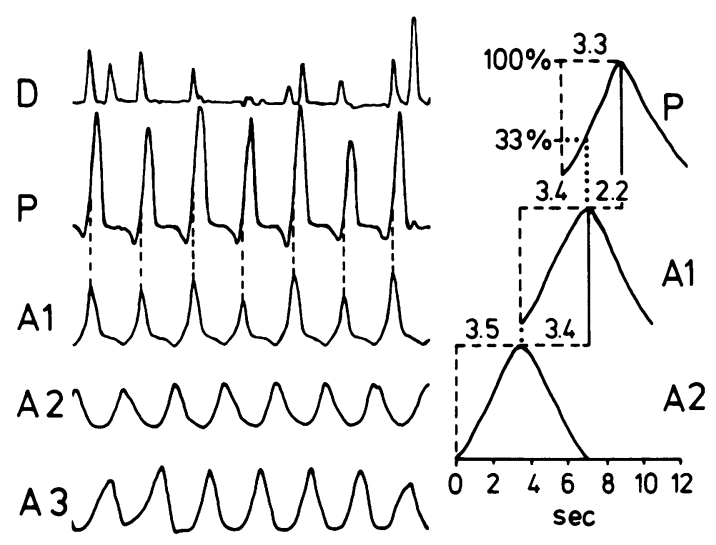

Fig. 5 Lefi panel: motility tracings of antrum, pyloric sphincter, and duodenum showing propagation of peristaltic waves. Right panel: schematic drawing of sequence of antral and pyloric contractions based on mean values of contractiontime and time-differences of contraction maxima of adjacent recording sites. Location of transducers as in Fig. 1.

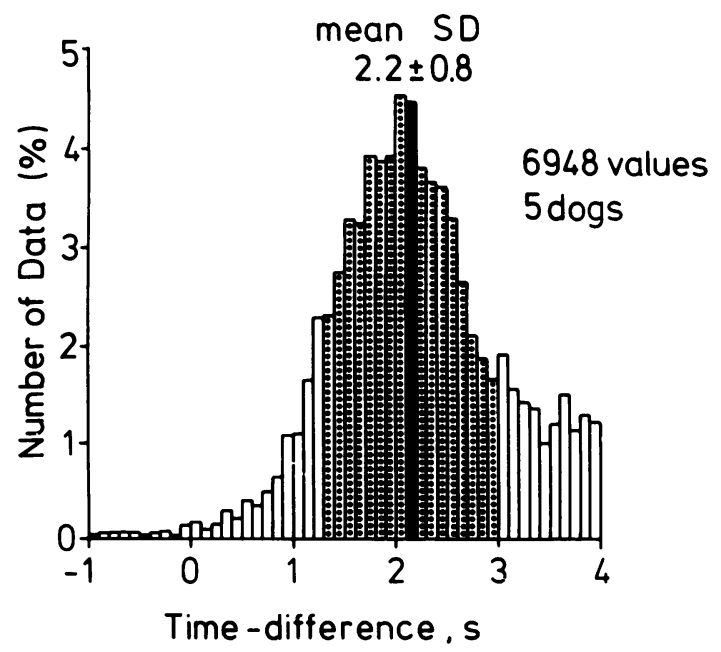

Fig. 6 Histogram of time-differences between contraction maxima of terminal antrum (site A1) and of pyloric sphincter with liquid meals and those of varying viscosity. experiments on the five dogs were summarised. The results are shown in Table 2 . The time-differences of the contraction maxima decreased and thus the velocity of the peristaltic wave increased during propagation from site $A 2$ of the antrum to the pyloric sphincter. The contraction maxima of site Al occurred $2.2 \pm 0.8$ seconds before the contraction maxima of the pyloric sphincter (Fig. 6). Thus no simultaneous contractions of the terminal antrum occurred. The mean contractiontime of the pyloric sphincter was $3.3 \pm 1.2 \mathrm{~s}$ (Table 2). When this value was related to the timedifferences of the contractions between site $A 1$ and the pyloric sphincter, it was found that the pyloric sphincter had reached $33 \%$ of its maximal contraction during the contraction maxima of site Al (Fig. 5, right panel).

\section{Discussion}

From experiments on dogs using plastic spheres as solid markers it was concluded that gastric emptying of solids is regulated by simultaneous contractions of the terminal antrum and the pyloric sphincter. ${ }^{8924}$ Simultaneous contractions of the terminal antrum were already seen in the isolated stomach of $\operatorname{dogs}^{25}$ and were confirmed by fluoroscopy in dogs ${ }^{10}$ and in man. ${ }^{11-13}$ These results, however, were based on radiographic findings and on records of the electric activity. Exact measurements of the mechanical activity of the terminal antrum and pyloric sphincter have not been done.

The present experiments have shown that the pyloric sphincter relaxed when the peristaltic wave travelled over the proximal antrum; the pylorus was open when the wave moved over the middle of the antrum, and it started to contract when the wave proceeded over the terminal antrum. Computer analysis of 6948 contractions of the terminal antrum revealed that contraction maxima of the terminal antrum were reached on average $2.2 \pm$ $0.8 \mathrm{~s}$ before the contraction maxima of the pyloric sphincter. Simultaneous contractions of the ter-

Table 2 Contraction-time and propagation of peristaltic waves with liquid meals and those of varying viscosity in five dogs

\begin{tabular}{lllllll}
\hline Site & $\begin{array}{l}\text { Contraction- } \\
\text { time } \\
(s)\end{array}$ & $\begin{array}{l}\text { Number of } \\
\text { contractions } \\
\text { analysed }\end{array}$ & $\begin{array}{l}\text { Propagation } \\
\text { from } \\
\text { site }\end{array}$ & $\begin{array}{l}\text { Time- } \\
\text { difference } \\
(\mathrm{s})\end{array}$ & $\begin{array}{l}\text { Velocity of } \\
\text { propagation } \\
(\mathrm{cm} / \mathrm{s})\end{array}$ & $\begin{array}{l}\text { Number of } \\
\text { contractions } \\
\text { analysed }\end{array}$ \\
\hline $\mathrm{A} 2$ & $3.5 \pm 1.5$ & 8656 & $\mathrm{~A} 2$ to A1 & $3.4 \pm 1.4$ & $0.8 \pm 0.8$ & 7250 \\
$\mathrm{~A} 1$ & $3.4 \pm 1.3$ & 8134 & A1 to P & $2.2 \pm 0.8$ & $1.1 \pm 0.8$ & 6948 \\
$\mathrm{P}$ & $3.3 \pm 1.2$ & 8139 & &
\end{tabular}

Data are means \pm SD.

Abbreviations as in Fig. 1. 
minal antrum and pyloric sphincter occurred very seldom. The controversy in the literature over the type of contraction of the terminal antrum seems to be a matter of interpretation, in that the quickly moving peristaltic wave is described as a simultaneous, concentric, or systolic contraction. ${ }^{11-13}$

The present experiments revealed that only sufficiently liquefied ingesta were delivered into the duodenum, whereas contents of medium and high viscosity were retropelled. This discriminatory function of the stomach was caused by adjusting the depth of the indentations of the antral waves to the viscosity of the gastric contents. The pylorus was involved in this regulation of gastric emptying more by its anatomical bottleneck than by its phasic contractions. The primary function of the pyloric contractions might be to clear the pylorus of obstructive material or to prevent duodenogastric reflux. ${ }^{16}$

We suggest that the depth of the peristaltic waves might be regulated by tension receptors in the gastric wall. Slowly adapting in-series tension receptors are the predominant mechanoreceptors of the stomach. ${ }^{26}{ }^{27}$ When the gastric contents are liquid, the peristaltic contractions produce a small increase in wall tension, as the resistance to the flow of liquids is low. The indentation of the peristaltic wave might thereby be deep. With viscous contents, however, resistance to the flow of the ingesta is great. The peristaltic contractions produce great increases in wall tension. By stimulation of tension receptors inhibitory intrinsic or extrinsic reflexes might be elicited, and the constriction of the same peristaltic wave might be diminished. In this way, the depth of the peristaltic indentation might be regulated in response to the viscosity of the gastric contents. This concept of regulation is in agreement with the results on the motility of the forestomachs in ruminants. Leek ${ }^{28}{ }^{29}$ found that the frequency and strength of the contractions of the forestomachs are influenced by a reflex mechanism originating from slowly adapting in-series tension receptors in the wall. This concept might also explain the differences between the recordings of strain gauge transducers and induction coils. Strain gauge transducers measure predominantly the isometric component of the contraction, whereas induction coils measure the isotonic component. When the resistance to the flow of the ingesta is low, the transfer of ingesta might depend predominantly on isotonic contractions. Thus, it is necessary to analyse both components of the contractions in order to get more information about the function of gastrointestinal motor activity.
The authors are grateful to Mrs Margrit Hartmann and Mrs Ingeborg Ehrlein for technical assistance, and to Dr Erich Hiesinger for help in computer analysis. This investigation was supported by the Deutsche Forschungsgemeinschaft, grant Eh 64/1.

\section{References}

1 Ehrlein HJ. Gastric motility and emptying in rabbits. In: Vantrappen G, ed. Proceedings of the fifth international symposium on gastro-intestinal motility. Herentals: Typoff-Press, 1975:284-9.

2 Reference deleted.

3 Heading RC, Tothill P, McLoughlin GP, Shearman DJG. Gastric emptying rate measurement in man. Gastroenterology 1976; 71:45-50.

4 Hinder RA, Kelly KA. Canine gastric emptying of solids and liquids. Am J Physiol 1977; 233:E335-40.

5 Malagelada JR. Quantification of gastric solid-liquid discrimination during digestion of ordinary meals. Gastroenterology 1977; 72:1246-67.

6 Martinez DA, Olsen HW. Simultaneous measurement of liquid and solid phase gastric emptying using a simple dual isotope technique. Gastroenterology 1978; 74:1135.

7 Meyer JH, Thompson JB, Cohen MB, Shadchehr A, Mandiola SA. Sieving of solid food by the canine stomach and sieving after gastric surgery. Gastroenterology 1979; 76:804-13.

8 Dozois RR, Kelly KA, Code CF. Effect of distal antrectomy on gastric emptying of liquids and solids. Gastroenterology 1971; 61:675-81.

9 Wilbur BG, Kelly KA. Effect of proximal gastric, complete gastric, and truncal vagotomy on canine gastric electric activity, motility, and emptying. Ann Surg 1973; 178:295-303.

10 Carlson HC, Code CF, Nelson RA. Motor action of the canine gastro-duodenal junction: a cineradiographic, pressure, and electric study. Am J Dig Dis 1966; 11:155-72.

11 Vicary F. La Motricité de l'Antre Gastrique. Paris: Editions Doin, 1967:81-93.

12 Keet AD. The prepyloric contractions in the normal stomach. Acta Radiol 1957; 48:413-24.

13 Keet AD. Diameter of the pyloric aperture in relation to the contraction of the canalis egestorius. Acta Radiol 1962; 57:31-44.

14 Cannon WB. The movements of the stomach studied by means of the roentgen rays. Am J Physiol 1898; 1:359--81.

15 Pröve J. Untersuchungen über den Mechanismus der Magenentleerung sowie den Einfluß von Pentagastrin, 5Hydroxytryptophan und Insulin auf die Magenentleerung und -motorik beim Hund. University of Hohenheim: Dissertion, 1979.

16 Ehrlein HJ, Pröve J, Schweiker W. The function of the pyloric sphincter for regulating gastric emptying and for preventing reflux in dogs. In: Christensen $\mathrm{J}$, ed. Gastrointestinal motility. New York: Raven Press, 1980:177-84.

17 Ehrlein HJ. Strain gauge transducers for recording gastrointestinal motility in unanesthetized animals. $Z$ Gastroenterol 1980; 18:191-7.

18 Brody DA, Quigley JP. Application of the 'inductograph' 
to the registration of movements, particularly of body structures such as the pyloric sphincter. J Lab Clin Med 1944; 29:863-7.

19 Louckes HS, Quigley JP, Kersey J. Inductograph method for recording muscle activity especially sphincter physiology. Am J Physiol 1960; 199:301-10.

20 Engelhardt W, Tolkmitt G, Ehrlein HJ, Stahlgren LH. Ein einfaches Gerät zur induktiven Messung der Darmmotilität mit implantierten Spulen. Pfluegers Arch 1967; 294:229-34.

21 Ehrlein HJ. A new technique for simultaneous radiography and recording of gastrointestinal motility in unanesthetized dogs. Lab Anim Sci 1980; 30:879-84.

22 Ehrlein HJ, Hiesinger E. Computer analysis of mechanical activity of gastroduodenal junction in unanesthetized dogs. $Q J$ Exp Physiol 1982; 67 (In press).

23 Sachs L. Statistische Auswertungsmethoden. Berlin, Heidelberg, New York: Springer, 1971:230-8.
24 Kelly KA. Canine gastric motility and emptying: electrical, neural and hormonal controls. In: Daniel EE, ed. Proceedings of the fourth international symposium on gastrointestinal motility. Vancouver: Mitchell Press, 1973:463-70.

25 Hofmeister F, Schuetz E. Über die automatischen Bewegungen des Magens. Arch Exp Pathol Pharmakol $1886 ; 20: 1-39$.

26 Iggo A. Tension receptors in the stomach and the urinary bladder. J Physiol 1955; 128:593-607.

27 Paintal AS. A study of gastric stretch receptors. Their role in the peripheral mechanism of satiation of hunger and thirst. J Physiol 1954; 126;255-70.

28 Leek BF. An electrophysiological investigation of gastric activity in the medulla of sheep. J Physiol 1968; 198:59-60P.

29 Leek BF. Reticulo-ruminal function and dysfunction. Vet $\operatorname{Rec} 1$ 1969; 84:238-43. 